\title{
Adhésion commune aux démarches qualité en médecine
}

\author{
Roxane Kübler ${ }^{\mathrm{a}}$, Esther Kraft ${ }^{\mathrm{b}}$ \\ a Responsable opérationnelle de I'Académie suisse pour la qualité en médecine (ASOM) de la FMH \\ ${ }^{b}$ Cheffe de la division Données, démographie et qualité (DDQ) de la FMH
}

\begin{abstract}
La charte qualité dont l'objectif est de développer des démarches qualité transparentes et obligatoires a été élaborée pendant deux ans par le Forum Qualité de l'ASQM. Après une consultation largement suivie au sein du corps médical, elle a été finalisée et signée par 57 organisations médicales.
\end{abstract}

\section{Contexte}

L’activité médicale est basée sur la responsabilité du médecin face à ses patients. La qualité de sa prestation fait naturellement partie de son travail et bénéficie aussi bien au patient et à ses proches qu'au médecin. De ce fait, il faut garantir et encourager les démarches qualité sous tous leurs aspects. C'est un point fondamental de l'identité médico-éthique de tout médecin, du Code de déontologie de la FMH et du droit suisse ${ }^{1}$. Les évolutions politiques et sociales actuelles en matière de santé exigent que l'évaluation des prestations médicales se base sur des critères axés sur la qualité. S'agissant des critères de qualité nationaux pour l'admission des médecins, la FMH a exigé de manière claire et répétée des compétences linguistiques obligatoires

1 Cf. art. 58, 59 et 59a LAMal et art. 77 OAMal.

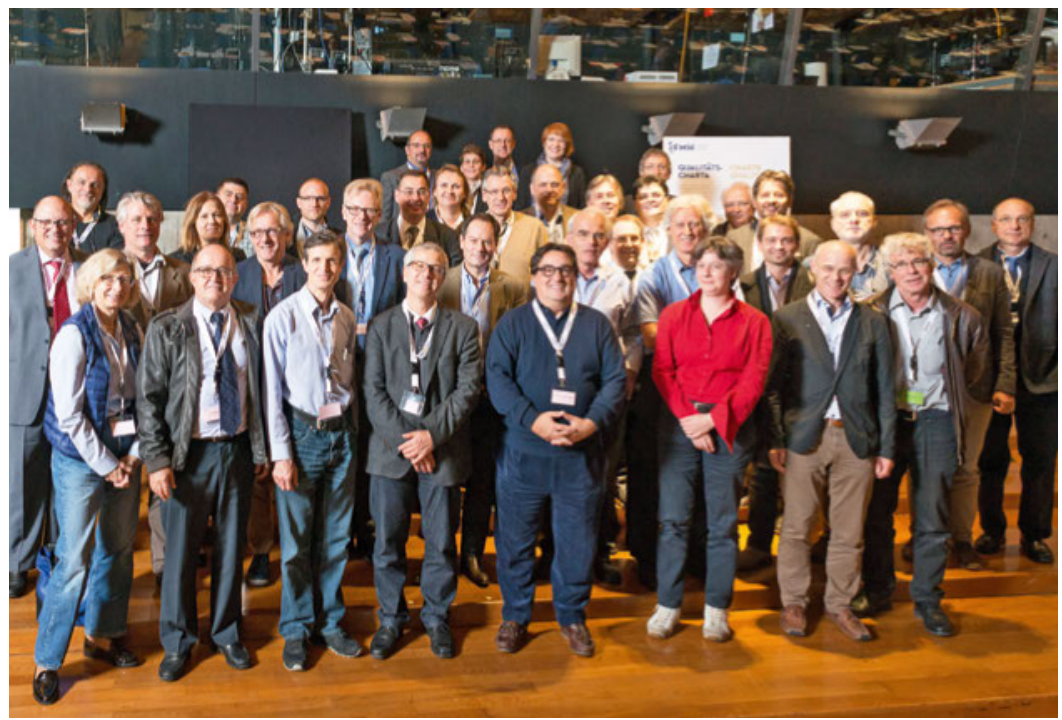

Signature solennelle lors de la Chambre médicale du 27 octobre 2016: les représentants des organisations signataires de la charte qualité de I'ASOM (source: Frederike Asael). de niveau B2 minimum assorties d'au moins trois ans d'activité dans un établissement de formation postgraduée suisse reconnu dans la discipline visée pour l'admission et d'au moins 5500 heures d'études de médecine. Les sociétés de discipline, les organisations cantonales de médecine et les sociétés faîtières sont appelées à développer et à garantir des stratégies qualité et à élaborer une attestation transparente de la qualité fournie.

\section{Elaboration}

Pendant deux ans, les délégués et les délégués suppléants du Forum Qualité de l'Académie suisse pour la qualité en médecine (ASQM) ont élaboré une charte qui, de janvier à avril 2016, a été mise en consultation auprès des organisations représentées à la Chambre médicale. En juin 2016, le Forum Qualité a approuvé la version finalisée de la charte qualité de l'ASQM que le Comité central de la FMH a ensuite adoptée. L'Assemblée des délégués de la FMH a été informée en septembre 2016 avant que toutes les organisations représentées à la Chambre médicale soient invitées à la signer. La signature symbolique de la charte a eu lieu dans le cadre de la Chambre médicale en octobre 2016.

\section{Objectifs}

La charte qualité de l'ASQM poursuit les objectifs suivants: les organisations médicales suisses

- rappellent conjointement leur adhésion aux principes fondamentaux de la qualité en matière de transparence, d'obligation, de durabilité/exactitude, de compétences et de ressources;

- acceptent des obligations claires, mais aussi transfèrent à l'externe des exigences communes envers 
Principes de base d'une charte de qualité

\begin{tabular}{|c|c|c|c|}
\hline Transparence & \multicolumn{2}{|c|}{ Caractère obligatoire } & Durabilité / Exactitude / Plus-value \\
\hline 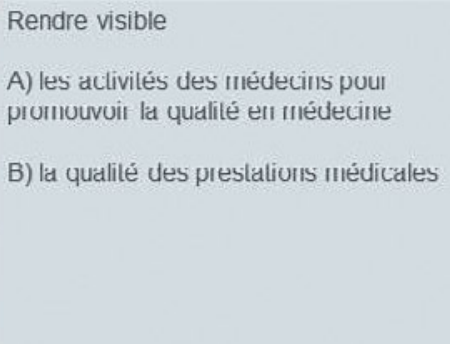 & \multicolumn{2}{|c|}{$\begin{array}{l}\text { Rédiyel } \\
\text { C) Une stratégie qualité } \\
\text { D) Un rapport sur la qualité }\end{array}$} & $\begin{array}{l}\text { Piendie ell cumıle } \\
\text { E) L'aspect durable } \\
\text { F) La formation pré-, postgraduée et } \\
\text { continue } \\
\text { G) Les bases légales en matière de } \\
\text { protection des données } \\
\text { H) La plus-value }\end{array}$ \\
\hline \multicolumn{3}{|c|}{ Compétences } & Ressources \\
\hline \multicolumn{2}{|c|}{$\begin{array}{l}\text { Le corps médical } \\
\text { l) se charge de la détinition, de l'assurance et du releve de la } \\
\text { qualité des prestations médicales. } \\
\text { J) est associé à toutes les activités qualité du milieu politique, } \\
\text { de l'administration et des organisations de santé, et } \\
\text { inversement. }\end{array}$} & \multicolumn{2}{|c|}{$\begin{array}{l}\text { K) Utilisation des synergies } \\
\text { L) Indemnisation des démarches en faveur de la qualité }\end{array}$} \\
\hline
\end{tabular}

Académie suisse pour la qualité en médecine ASQM

M) L'ASQM promeut la cohésion et le transfert d'informations en matière de qualité au sein du corps médical.

N) L'ASQM réceptionne et coordonne toutes les questions de partenaires des milieux politiques, de l'administration et du domaine de la santé concernant la qualité des soins.

O) L'ASQM associe aussi bien les organisations médicales concernées que les organisations partenaires dans le cadre de ses propres projets afin de garantir suffisamment tôt un ancrage spécialisé et politique.

Figure 1: Les 15 principes fondamentaux de la charte qualité de I'ASOM (source: DDQ/ASOM).

le monde politique, l'administration, et d'autres organisations médicales;

- introduisent une obligation notamment en ce qui concerne la transparence en matière de qualité des prestations médicales.

\section{Première signature}

Lors de la Chambre médicale du 27 octobre 2016, le Dr Christoph Bosshard a fait le point sur la charte qualité de l'ASQM. Il en a profité pour souligner l'importance pour les organisations médicales de rappeler ensemble leur adhésion au caractère obligatoire et à la transparence des démarches qualité en médecine. Il a lu le nom des organisations signataires et remercié les présidentes et présidents pour leur engagement avant de les inviter à la signature symbolique de la charte. Les 57 organisations suivantes ont déjà signé la charte qualité de l'ASQM (liste au 27 octobre 2016):

- ASMAC

- Association suisse des médecins indépendants travaillant en cliniques privées et hôpitaux

- Collège de médecine de premier recours

- FMCH
- FMP

- FMPP

- Médecins de famille et de l'enfance Suisse

- Ordine dei Medici del Cantone Ticino

- SFSM

- Société de médecine du canton d'Unterwald

- Société de médecine du canton de Bâle-Campagne

- Société de médecine du canton de Lucerne

- Société de médecine du canton de Saint-Gall

- Société de médecine du canton de Schwytz

- Société de médecine du canton de Soleure

- Société de médecine du canton de Thurgovie

- Société de médecine du canton de Zurich

- Société médicale du Valais

- Société neuchâteloise de médecine

- Société suisse d'allergologie et d'immunologie

- Société suisse d'infectiologie

- Société suisse d'oncologie médicale

- Société suisse d'ophtalmologie

- Société suisse d'oto-rhino-laryngologie et de chirurgie cervico-faciale

- Société suisse d'urologie

- Société suisse d'anesthésiologie et de réanimation

- Société suisse de cardiologie 
- Société suisse de chirurgie

- Société suisse de chirurgie cardiaque et vasculaire thoracique

- Société suisse de chirurgie de la main

- Société suisse de chirurgie plastique, reconstructive et esthétique

- Société suisse de chirurgie thoracique

- Société suisse de chirurgie vasculaire

- Société suisse de dermatologie et vénéréologie

- Société suisse de gastroentérologie

- Société suisse de génétique médicale

- Société suisse de gynécologie et d'obstétrique

- Société suisse de médecine du travail

- Société suisse de médecine d'urgence et de sauvetage

- Société suisse de médecine interne générale

- Société suisse de médecine légale

- Société suisse de médecine manuelle

- Société suisse de médecine nucléaire

- Société suisse de médecine tropicale et de médecine des voyages

- Société suisse de neurochirurgie

- Société suisse de neurologie

- Société suisse de pathologie
- Société suisse de pédiatrie

- Société suisse de pharmacologie et de toxicologie clinique

- Société suisse de pneumologie

- Société suisse de psychiatrie et psychothérapie de l'enfant et de l'adolescent

- Société suisse de psychiatrie et psychothérapie

- Société suisse de radiologie

- Société suisse de radio-oncologie

- Société suisse des médecins-conseils et médecins d'assurances

- Swiss Orthopaedics

- Union des sociétés suisses de médecine complémentaire

La signature de la charte qualité reste facultative et il est toujours possible de la signer à tout moment après la Chambre médicale. Si vous le souhaitez, n'hésitez pas à nous contacter, le mieux par courriel à asqm[at]fmh.ch.

Sur le site www.asqm.ch $\rightarrow$ Charte qualité, vous trouverez de plus amples informations concernant la charte qualité de l'ASOM mais aussi des modèles de stratégie qualité et de rapport qualité, ainsi qu'une galerie photos de la signature de la charte lors de la Chambre médicale.

La Société suisse d'oncologie médicale a été la première organisation médicale à avoir signé la charte qualité de I'ASOM. Trois questions à son président, le Prof. Markus Maximilian Borner:

Quelle a été la motivation principale de votre société à signer la charte qualité de l'ASOM?

La qualité des soins oncologiques en Suisse est très élevée, comme le montrent les comparaisons avec l'étranger - nous le devons aux conditions de notre formation postgraduée et continue structurée. De nos jours, la tendance va cependant vers une institutionnalisation de la qualité, ce à quoi contribue la charte qualité. Une question aussi importante que celle-ci devrait être abordée de manière centralisée également pour des raisons économiques au lieu d'être reléguée au maillon le plus faible de la chaîne de soins.

Fédération des

médecins suisses (FMH)

Division DDQ

Elfenstrasse 18

Case postale 300

CH-3000 Berne 15

roxane.kuebler[at]fmh.ch

Tél. 0313591111 nationales sur le résultat. Je veux parler ici de recherche sur les soins, qu'il s'agit de mettre en lien avec les questions de qualité, ce qui représente un énorme défi mais également une grande opportunité.

Où voyez-vous les chances de la charte qualité de I'ASOM?

Si la FMH parvient à mettre en place des instruments simples et fondés dans le domaine des registres, nous disposerons ainsi des outils nécessaires pour améliorer continuellement les soins avec les paramètres de notre choix. Un point particulièrement important à relever est le fait que les auteurs de la charte abordent également la question de la charge de travail supplémentaire et de sa rétribution - c'est là une condition sine qua non pour les fournisseurs de prestations dans toutes les disciplines. 